\title{
Complete Fairness in Multi-party Computation without an Honest Majority
}

\author{
S. Dov Gordon and Jonathan Katz \\ Dept. of Computer Science, University of Maryland
}

\begin{abstract}
Gordon et al. recently showed that certain (non-trivial) functions can be computed with complete fairness in the two-party setting. Motivated by their results, we initiate a study of complete fairness in the multi-party case and demonstrate the first completely-fair protocols for non-trivial functions in this setting. We also provide evidence that achieving fairness is "harder" in the multi-party setting, at least with regard to round complexity.
\end{abstract}

\section{Introduction}

In the setting of secure computation, a group of parties wish to run a protocol for computing some function of their inputs while preserving, to the extent possible, security properties such as privacy, correctness, input independence and others. These requirements are formalized by comparing a real-world execution of the protocol to an ideal world where there is a trusted entity who performs the computation on behalf of the parties. Informally, a protocol is "secure" if for any real-world adversary $\mathcal{A}$ there exists a corresponding ideal-world adversary $\mathcal{S}$ (corrupting the same parties as $\mathcal{A}$ ) such that the result of executing the protocol in the real world with $\mathcal{A}$ is computationally indistinguishable from the result of computing the function in the ideal world with $\mathcal{S}$.

One desirable property is fairness which, intuitively, means that either everyone receives the output, or else no one does. Unfortunately, it has been shown by Cleve 1 that complete fairness is impossible in general without a majority of honest parties. Until recently, Cleve's result was interpreted to mean that no non-trivial functions could be computed with complete fairness without an honest majority. A recent result of Gordon et al. 2., however, shows that this folklore is wrong; there exist non-trivial functions than can be computed with complete fairness in the two-party setting. Their work demands that we re-evaluate our current understanding of fairness.

Gordon et al. 2, deal exclusively with the case of two-party computation, and leave open the question of fairness in the multi-party setting. Their work does not immediately extend to the case of more than two parties. (See also the discussion in the section that follows.) An additional difficulty that arises in the

The original version of this chapter was revised: The copyright line was incorrect. This has been corrected. The Erratum to this chapter is available at DOI: 10.1007/978-3-642-00457-5_36

* This work was supported by NSF CNS-0447075, NSF CCF-0830464, and US-Israel

Binational Science Foundation grant \#2004240.

O. Reingold (Ed.): TCC 2009, LNCS 5444, pp. 19-35, 2009.

(C) Springer-Verlag Berlin Heidelberg 2009 
multi-party setting is the need to ensure consistency between the outputs of the honest parties, even after a malicious abort. In more detail: in the two-party setting, it suffices for an honest party's output (following an abort by the other party) to be consistent only with its own local input. But in the multi-party setting, the honest parties' outputs must each be consistent with all of their inputs. This issue is compounded by the adversary's ability to adaptively abort the $t$ malicious players in any order and at any time, making fairness in the multi-party setting even harder to achieve.

We initiate a study of complete fairness in the multi-party setting. We focus on the case when a private 1 broadcast channel (or, equivalently, a PKI) is available to the parties; note that Cleve's impossibility result applies in this case as well. Although one can meaningfully study fairness in the absence of broadcast, we have chosen to assume broadcast so as to separate the question of fairness from the question of agreement (which has already been well studied in the distributed systems literature). Also, although the question of fairness becomes interesting as soon as an honest majority is not assumed, here we only consider the case of completely-fair protocols tolerating an arbitrary number of corrupted parties. We emphasize that, as in 2, we are interested in obtaining complete fairness rather than some notion of partial fairness.

\subsection{Our Results}

A natural first question is whether two-party feasibility results 2 can be extended "easily" to the multi-party setting. More formally, say we have a function $f:\{0,1\} \times \cdots \times\{0,1\} \rightarrow\{0,1\}$ taking $n$ boolean inputs. (We restrict to boolean inputs/outputs for simplicity only.) For any non-trivial subset $I \subset[n]$, define the partition $f_{I}$ of $f$ as the two-input function $f_{I}:\{0,1\}^{|I|} \times\{0,1\}^{n-|I|} \rightarrow\{0,1\}$ given by

$$
f_{I}(y, z)=f(x),
$$

where $x \in\{0,1\}^{n}$ is such that $x_{I}=y$ and $x_{\bar{I}}=z$. It is not hard to see that if there exists an $I$ for which $f_{I}$ cannot be computed with complete fairness in the two-party setting, then $f$ cannot be computed with complete fairness in the multi-party setting. Similarly, the round complexity for computing $f$ with complete fairness in the multi-party case must be at least the round complexity of fairly computing each $f_{I}$. What about the converse? We show the following negative result regarding such a "partition-based" approach to the problem:

Theorem 1. (Under suitable cryptographic assumptions) there exists a 3-party function $f$ all of whose partitions can be computed with complete fairness in $\mathcal{O}(1)$ rounds, but for which any multi-party protocol computing $f$ with complete fairness requires $\omega(\log k)$ rounds, where $k$ is the security parameter.

This indicates that fairness in the multi-party setting is qualitatively harder than fairness in the two-party setting. (A somewhat analogous result in a different context was shown by Chor et al. 3 .)

${ }^{1}$ We assume private broadcast so as to ensure security against passive eavesdroppers (who do not corrupt any parties). 
The function $f$ for which we prove the above theorem is interesting in its own right: it is the 3 -party majority function (i.e., voting). Although the $\omega(\log k)$ round lower bound may seem discouraging, we are able to show a positive result for this function; to the best of our knowledge, this represents the first non-trivial feasibility result for complete fairness in the multi-party setting.

Theorem 2. (Under suitable cryptographic assumptions) there exists an $\omega(\log k)$-round protocol for securely computing 3-party majority with complete fairness.

Our efforts to extend the above result to $n$-party majority have been unsuccessful. One may therefore wonder whether there exists any (non-trivial) function that can be computed with complete fairness for general $n$. Indeed, there is:

Theorem 3. (Under suitable cryptographic assumptions) for any number of parties $n$, there exists an $\Theta(n)$-round protocol for securely computing boolean OR with complete fairness.

OR is non-trivial in our context: OR is complete for multi-party computation (without fairness) 4], and cannot be computed with information-theoretic privacy even in the two-party setting [5].

Relation to prior work. At a superficial level, the proof of the $\omega(\log k)$-round lower bound of Theorem 1 uses an approach similar to that used to prove an analogous lower bound in [2]. We stress, however, that our theorem does not follow as a corollary of that work (indeed, it cannot since each of the partitions of $f$ can be computed with complete fairness in $\mathcal{O}(1)$ rounds). We introduce new ideas to prove the result in our setting; in particular, we rely in an essential way on the fact that the output of any two honest parties must agree (whereas this issue does not arise in the two-party setting considered in [2).

Ishai et al. 6] propose a protocol that is resilient to a dishonest majority in a weaker sense than that considered here. Specifically, their protocol achieves the following guarantee (informally): when $t<n$ parties are corrupted then a real execution of the protocol is as secure as an execution in the ideal world with complete fairness where the adversary can query the ideal functionality $\mathcal{O}(t)$ times (using different inputs each time). While this definition may guarantee privacy for certain functions (e.g., for the sum function), it does not prevent the malicious parties from biasing the output of the honest parties. We refer the reader to their work for further discussion.

\subsection{Outline of the Paper}

We include the standard definitions of secure multi-party computation in the full version of this paper [7. We stress that although the definitions are standard, what is not standard is that we are interested in attaining complete fairness even though we do not have an honest majority. 
We begin with our negative result, showing that any completely-fair protocol for 3-party majority requires $\omega(\log k)$ rounds. Recall that what is especially interesting about this result is that it demonstrates a gap between the round complexities required for completely-fair computation of a function and its (two-party) partitions. In Section [3, we show an $\omega(\log k)$-round protocol for completely-fair computation of 3-party majority. In Section 4 we describe our feasibility result for the case of boolean OR.

\section{A Lower Bound on the Round Complexity of Majority}

\subsection{Proof Overview}

In this section, we prove Theorem 1 taking as our function $f$ the three-party majority function maj. That is, $\operatorname{maj}\left(x_{1}, x_{2}, x_{3}\right)=0$ if at least two of the three values $\left\{x_{1}, x_{2}, x_{3}\right\}$ are 0 , and is 1 otherwise. Note that any partition of maj is just (isomorphic to) the greater-than-or-equal-to function, where the domain of one input can be viewed as $\{0,1,2\}$ and the domain of the other input can be viewed as $\{0,1\}$ (in each case, representing the number of ' 1 ' inputs held). Gordon et al. 2] show that, under suitable cryptographic assumptions, the greater-thanor-equal-to function on constant-size domains can be securely computed with complete fairness in $\mathcal{O}(1)$ rounds.

We prove Theorem 1 by showing that any completely-fair 3-party protocol for maj requires $\omega(\log k)$ rounds. The basic approach is to argue that if $\Pi$ is any protocol for securely computing maj, then eliminating the last round of $\Pi$ results in a protocol $\Pi^{\prime}$ that still computes maj correctly "with high probability". Specifically, if the error probability in $\Pi$ is at most $\mu$ (that we will eventually set to some negligible function of $k$ ), then the error probability in $\Pi^{\prime}$ is at most $c \cdot \mu$ for some constant $c$. If the original protocol $\Pi$ has $r=\mathcal{O}(\log k)$ rounds, then applying this argument inductively $r$ times gives a protocol that computes maj correctly on all inputs with probability significantly better than guessing without any interaction at all. This gives the desired contradiction.

To prove that eliminating the last round of $\Pi$ cannot affect correctness "too much", we consider a constraint that holds for the ideal-world evaluation of maj. (Recall, we are working in the ideal world where complete fairness holds.) Consider an adversary who corrupts two parties, and let the input of the honest party $P$ be chosen uniformly at random. The adversary can learn $P$ 's input by submitting $(0,1)$ or $(1,0)$ to the trusted party. The adversary can also try to bias the output of maj to be the opposite of $P$ 's choice by submitting $(0,0)$ or $(1,1)$; this will succeed in biasing the result half the time. But the adversary cannot both learn $P$ 's input and simultaneously bias the result. (If the adversary submits $(0,1)$ or $(1,0)$, the output of maj is always equal to $P$ 's input; if the adversary submits $(0,0)$ or $(1,1)$ the the output of maj reveals nothing about $P$ 's input.) Concretely, for any ideal-world adversary the sum of the probability that the adversary guesses $P$ 's input and the probability that the output of maj is not equal to $P$ 's input is at most 1 . In our proof, we show that if correctness 
holds with significantly lower probability when the last round of $\Pi$ is eliminated, then there exists a real-world adversary violating this constraint.

\subsection{Proof Details}

We number the parties $P_{1}, P_{2}, P_{3}$, and work modulo 3 in the subscript. The input of $P_{j}$ is denoted by $x_{j}$. The following claim formalizes the ideal-world constraint described informally above.

Claim 4. For all $j \in\{1,2,3\}$ and any adversary $\mathcal{A}$ corrupting $P_{j-1}$ and $P_{j+1}$ in an ideal-world computation of maj, we have

$$
\operatorname{Pr}\left[\mathcal{A} \text { correctly guesses } x_{j}\right]+\operatorname{Pr}\left[\operatorname{OutPuT}_{j} \neq x_{j}\right] \leq 1,
$$

where the probabilities are taken over the random coins of $\mathcal{A}$ and random choice of $x_{j} \in\{0,1\}$.

Proof. Consider an execution in the ideal world, where $P_{j}$ 's input $x_{j}$ is chosen uniformly at random. Let EQUAL be the event that $\mathcal{A}$ submits two equal inputs (i.e., $x_{j-1}=x_{j+1}$ ) to the trusted party. In this case, $\mathcal{A}$ learns nothing about $P_{j}$ 's input and so can guess $x_{j}$ with probability at most $1 / 2$. It follows that:

$$
\operatorname{Pr}\left[\mathcal{A} \text { correctly guesses } x_{j}\right] \leq \frac{1}{2} \operatorname{Pr}[\text { EQUAL }]+\operatorname{Pr}[\overline{\text { EQUAL }}] .
$$

Moreover, $\operatorname{Pr}\left[\right.$ OUTPUT $\left._{j} \neq x_{j}\right]=\frac{1}{2} \operatorname{Pr}[\mathrm{EQUAL}]$ since OUTPUT $_{j} \neq x_{j}$ occurs only if $\mathcal{A}$ submits $x_{j-1}=x_{j+1}=\bar{x}_{j}$ to the trusted party. Therefore:

$$
\begin{aligned}
& \operatorname{Pr}\left[\mathcal{A} \text { correctly guesses } x_{j}\right]+\operatorname{Pr}\left[\text { OUTPUT }_{j} \neq x_{j}\right] \\
& \quad \leq \frac{1}{2} \operatorname{Pr}[\text { EQUAL }]+\operatorname{Pr}[\overline{\text { EQUAL }}]+\frac{1}{2} \operatorname{Pr}[\text { EQUAL }] \\
& \quad=\operatorname{Pr}[\text { EQUAL }]+\operatorname{Pr}[\overline{\text { EQUAL }}]=1,
\end{aligned}
$$

proving the claim.

Let $\Pi$ be a protocol that securely computes maj using $r=r(k)$ rounds. Consider an execution of $\Pi$ in which all parties run the protocol honestly except for possibly aborting in some round. We denote by $b_{j}^{(i)}$ the value that $P_{j-1}$ and $P_{j+1}$ both ${ }^{2}$ output if $P_{j}$ aborts the protocol after sending its round- $i$ message (and then $P_{j-1}$ and $P_{j+1}$ honestly run the protocol to completion). Similarly, we denote by $b_{j-1}^{(i)}$ (resp., $b_{j+1}^{(i)}$ ) the value output by $P_{j}$ and $P_{j+1}$ (resp., $P_{j}$ and $P_{j-1}$ ) when $P_{j-1}$ (resp., $P_{j+1}$ ) aborts after sending its round- $i$ message. Note that an adversary who corrupts, e.g., both $P_{j-1}$ and $P_{j+1}$ can compute $b_{j}^{(i)}$ immediately after receiving the round $i$ message of $P_{j}$.

${ }^{2}$ Security of $\Pi$ implies that the outputs of $P_{j-1}$ and $P_{j+1}$ in this case must be equal with all but negligible probability. For simplicity we assume this to hold with probability 1 but our proof can be modified easily to remove this assumption. 
Since $\Pi$ securely computes maj with complete fairness, the ideal-world constraint from the previous claim implies that for all $j \in\{1,2,3\}$, any inverse polynomial $\mu(k)$, and any poly-time adversary $\mathcal{A}$ controlling players $P_{j-1}$ and $P_{j+1}$, we have:

$$
\underset{x_{j} \leftarrow\{0,1\}}{\operatorname{Pr}}\left[\mathcal{A} \text { correctly guesses } x_{j}\right]+\underset{x_{j} \leftarrow\{0,1\}}{\operatorname{Pr}}\left[\operatorname{OUTPUT}_{j} \neq x_{j}\right] \leq 1+\mu(k)
$$

for $k$ sufficiently large. Security of $\Pi$ also guarantees that if the inputs of the honest parties agree, then with all but negligible probability their output must be their common input regardless of when a malicious $P_{j}$ aborts. That is, for $k$ large enough we have

$$
x_{j+1}=x_{j-1} \Rightarrow \operatorname{Pr}\left[b_{j}^{(i)}=x_{j+1}=x_{j-1}\right] \geq 1-\mu(k)
$$

for all $j \in\{1,2,3\}$ and all $i \in\{0, \ldots, r(k)\}$.

The following claim represents the key step in our lower bound.

Claim 5. Fix a protocol $\Pi$, a function $\mu$, and a value $k$ such that Equations (1) and (2) hold, and let $\mu=\mu(k)$. Say there exists an $i$, with $1 \leq i \leq r(k)$, such that for all $j \in\{1,2,3\}$ and all $c_{1}, c_{2}, c_{3} \in\{0,1\}$ it holds that:

$$
\operatorname{Pr}\left[b_{j}^{(i)}=\operatorname{maj}\left(c_{1}, c_{2}, c_{3}\right) \mid\left(x_{1}, x_{2}, x_{3}\right)=\left(c_{1}, c_{2}, c_{3}\right)\right] \geq 1-\mu .
$$

Then for all $j \in\{1,2,3\}$ and all $c_{1}, c_{2}, c_{3} \in\{0,1\}$ it holds that:

$$
\operatorname{Pr}\left[b_{j}^{(i-1)}=\operatorname{maj}\left(c_{1}, c_{2}, c_{3}\right) \mid\left(x_{1}, x_{2}, x_{3}\right)=\left(c_{1}, c_{2}, c_{3}\right)\right] \geq 1-5 \mu .
$$

Proof. When $j=1$ and $c_{2}=c_{3}$, the desired result follows from Equation (21); this is similarly true for $j=2, c_{1}=c_{3}$ as well as $j=3, c_{1}=c_{2}$.

Consider the real-world adversary $\mathcal{A}$ that corrupts $P_{1}$ and $P_{3}$ and sets $x_{1}=0$ and $x_{3}=1$. Then:

- $\mathcal{A}$ runs the protocol honestly until it receives the round- $i$ message from $P_{2}$.

- $\mathcal{A}$ then locally computes the value of $b_{2}^{(i)}$.

- If $b_{2}^{(i)}=0$, then $\mathcal{A}$ aborts $P_{1}$ without sending its round-i message and runs the protocol (honestly) on behalf of $P_{3}$ until the end. By definition, the output of $P_{2}$ will be $b_{1}^{(i-1)}$.

- If $b_{2}^{(i)}=1$, then $\mathcal{A}$ aborts $P_{3}$ without sending its round-i message and runs the protocol (honestly) on behalf of $P_{1}$ until the end. By definition, the output of $P_{2}$ will be $b_{3}^{(i-1)}$.

- After completion of the protocol, $\mathcal{A}$ outputs $b_{2}^{(i)}$ as its guess for the input of $P_{2}$.

Consider an experiment in which the input $x_{2}$ of $P_{2}$ is chosen uniformly at random, and then $\mathcal{A}$ runs protocol $\Pi$ with $P_{2}$. Using Equation (3), we have:

$$
\begin{aligned}
\operatorname{Pr}\left[\mathcal{A} \text { correctly guesses } x_{2}\right] & =\operatorname{Pr}\left[b_{2}^{(i)}=x_{2}\right] \\
& =\operatorname{Pr}\left[b_{2}^{(i)}=f\left(0, x_{2}, 1\right)\right] \geq 1-\mu .
\end{aligned}
$$


We also have:

$$
\begin{aligned}
\operatorname{Pr}\left[\text { Output }_{2} \neq x_{2}\right]= & \frac{1}{2} \cdot \operatorname{Pr}\left[\text { OutPuT }_{2}=1 \mid\left(x_{1}, x_{2}, x_{3}\right)=(0,0,1)\right] \\
& +\frac{1}{2} \cdot \operatorname{Pr}\left[\text { OutPuT }_{2}=0 \mid\left(x_{1}, x_{2}, x_{3}\right)=(0,1,1)\right] \\
= & \frac{1}{2}\left(\operatorname{Pr}\left[b_{1}^{(i-1)}=1 \wedge b_{2}^{(i)}=0 \mid\left(x_{1}, x_{2}, x_{3}\right)=(0,0,1)\right]\right. \\
& +\operatorname{Pr}\left[b_{3}^{(i-1)}=1 \wedge b_{2}^{(i)}=1 \mid\left(x_{1}, x_{2}, x_{3}\right)=(0,0,1)\right] \\
& +\operatorname{Pr}\left[b_{3}^{(i-1)}=0 \wedge b_{2}^{(i)}=1 \mid\left(x_{1}, x_{2}, x_{3}\right)=(0,1,1)\right] \\
& \left.+\operatorname{Pr}\left[b_{1}^{(i-1)}=0 \wedge b_{2}^{(i)}=0 \mid\left(x_{1}, x_{2}, x_{3}\right)=(0,1,1)\right]\right) .
\end{aligned}
$$

From Equation (11), we know that the sum of Equations (15) and (6) is upperbounded by $1+\mu$. Looking at the first summand in Equation (6), this implies that

$$
\operatorname{Pr}\left[b_{1}^{(i-1)}=1 \wedge b_{2}^{(i)}=0 \mid\left(x_{1}, x_{2}, x_{3}\right)=(0,0,1)\right] \leq 4 \mu .
$$

Probabilistic manipulation gives

$$
\begin{aligned}
& \operatorname{Pr}\left[b_{1}^{(i-1)}=1 \wedge b_{2}^{(i)}=0 \mid\left(x_{1}, x_{2}, x_{3}\right)=(0,0,1)\right] \\
& =1-\operatorname{Pr}\left[b_{1}^{(i-1)}=0 \vee b_{2}^{(i)}=1 \mid\left(x_{1}, x_{2}, x_{3}\right)=(0,0,1)\right] \\
& \geq 1-\operatorname{Pr}\left[b_{1}^{(i-1)}=0 \mid\left(x_{1}, x_{2}, x_{3}\right)=(0,0,1)\right]-\operatorname{Pr}\left[b_{2}^{(i)}=1 \mid\left(x_{1}, x_{2}, x_{3}\right)=(0,0,1)\right] \\
& \geq 1-\operatorname{Pr}\left[b_{1}^{(i-1)}=0 \mid\left(x_{1}, x_{2}, x_{3}\right)=(0,0,1)\right]-\mu,
\end{aligned}
$$

where the last inequality is due to the assumption of the claim. Combined with Equation (17), this implies:

$$
\operatorname{Pr}\left[b_{1}^{(i-1)}=0 \mid\left(x_{1}, x_{2}, x_{3}\right)=(0,0,1)\right] \geq 1-5 \mu .
$$

Applying an analogous argument starting with the third summand in Equation (6) gives

$$
\operatorname{Pr}\left[b_{3}^{(i-1)}=1 \mid\left(x_{1}, x_{2}, x_{3}\right)=(0,1,1)\right] \geq 1-5 \mu .
$$

Repeating the entire argument, but modifying the adversary to consider all possible pairs of corrupted parties and all possible settings of their inputs, completes the proof of the claim.

Theorem 6. Any protocol $\Pi$ that securely computes maj with complete fairness (assuming one exist 3 at all) requires $\omega(\log k)$ rounds.

\footnotetext{
${ }^{3}$ In the following section we show that such a protocol does, indeed, exist.
} 
Proof. Assume there exists a protocol $\Pi$ that securely computes maj with complete fairness using $r=\mathcal{O}(\log k)$ rounds. Let $\mu(k)=\frac{1}{4 \cdot 5^{r(k)}}$, and note that $\mu$ is noticeable. By the assumed security of $\Pi$, the conditions of Claim 5 hold for $k$ large enough; Equation (3), in particular, holds for $i=r(k)$. Fixing this $k$ and applying the claim iteratively $r(k)$ times, we conclude that $P_{j-1}$ and $P_{j+1}$ can correctly compute the value of the function, on all inputs, with probability at least $3 / 4$ without interacting with $P_{j}$ at all. This is clearly impossible.

\section{Fair Computation of Majority for Three Players}

In this section we describe a completely-fair protocol for computing maj for the case of $n=3$ parties. The high-level structure of our protocol is as follows: the protocol consists of two phases. In the first phase, the parties run a securewith-abort protocol to generate (authenticated) shares of certain values; in the second phase some of these shares are exchanged, round-by-round, for a total of $m$ iterations. A more detailed description of the protocol follows.

In the first phase of the protocol the parties run a protocol $\pi$ implementing a functionality ShareGen that computes certain values and then distributes authenticated 3-out-of-3 shares of these values to the parties. (See Fig. 11) Three sets of values $\left\{b_{1}^{(i)}\right\}_{i=0}^{m},\left\{b_{2}^{(i)}\right\}_{i=0}^{m}$, and $\left\{b_{3}^{(i)}\right\}_{i=0}^{m}$ are computed; looking ahead, $b_{j}^{(i)}$ denotes the value that parties $P_{j-1}$ and $P_{j+1}$ are supposed to output in case party $P_{j}$ aborts after iteration $i$ of the second phase; see below. The values $b_{j}^{(i)}$ are computed probabilistically, in the same manner as in 2 . That is, a round $i^{*}$ is first chosen according to a geometric distribution with parameter $\alpha=1 / 54$ (We will set $m$ so that $i^{*} \leq m$ with all but negligible probability.) Then, for $i<i^{*}$ the value of $b_{j}^{(i)}$ is computed using the true inputs of $P_{j-1}$ and $P_{j+1}$ but a random input for $P_{j}$; for $i \geq i^{*}$ the value $b_{j}^{(i)}$ is set equal to the correct output (i.e., it is computed using the true inputs of all parties). Note that even an adversary who knows all the parties' inputs and learns, sequentially, the values (say) $b_{1}^{(1)}, b_{1}^{(2)}, \ldots$ cannot determine definitively when round $i^{*}$ occurs.

We choose the protocol $\pi$ computing ShareGen to be secure-with-designatedabort [8] for $P_{1}$. Roughly speaking, this means privacy and correctness are ensured no matter what, and output delivery and (complete) fairness are guaranteed unless $P_{1}$ is corrupted; a formal definition in given in the full version [7].

The second phase of the protocol proceeds in a sequence of $m=\omega(\log n)$ iterations. (See Fig. 2]) In each iteration $i$, each party $P_{j}$ broadcasts its share of $b_{j}^{(i)}$. (We stress that we allow rushing, and do not assume synchronous broadcast.) Observe that, after this is done, parties $P_{j-1}$ and $P_{j+1}$ jointly have enough information to reconstruct $b_{j}^{(i)}$, but neither party has any information about $b_{j}^{(i)}$ on its own. If all parties behave honestly until the end of the protocol, then in the final iteration all parties reconstruct $b_{1}^{(m)}$ and output this value.

\footnotetext{
${ }^{4}$ This is the distribution on $\mathbb{N}=\{1,2, \ldots\}$ given by flipping a biased coin (that is
} heads with probability $\alpha$ ) until the first head appears. 


\section{ShareGen}

Inputs: Let the inputs to ShareGen be $x_{1}, x_{2}, x_{3} \in\{0,1\}$. (If one of the received inputs is not in the correct domain, then a default value of 1 is used for that player.) The security parameter is $k$.

\section{Computation:}

1. Define values $b_{1}^{(1)}, \ldots, b_{1}^{(m)}, b_{2}^{(1)}, \ldots, b_{2}^{(m)}$ and $b_{3}^{(1)}, \ldots, b_{3}^{(m)}$ in the following way:

- Choose $i^{*} \geq 1$ according to a geometric distribution with parameter $\alpha=1 / 5$ (see text).

- For $i=0$ to $i^{*}-1$ and $j \in\{1,2,3\}$ do:

- Choose $\hat{x}_{j} \leftarrow\{0,1\}$ at random.

- Set $b_{j}^{(i)}=\operatorname{maj}\left(x_{j-1}, \hat{x}_{j}, x_{j+1}\right)$.

- For $i=i^{*}$ to $m$ and $j \in\{1,2,3\}$, set $b_{j}^{(i)}=\operatorname{maj}\left(x_{1}, x_{2}, x_{3}\right)$.

2. For $0 \leq i \leq m$ and $j \in\{1,2,3\}$, choose $b_{j \mid 1}^{(i)}, b_{j \mid 2}^{(i)}$ and $b_{j \mid 3}^{(i)}$ as random threeway shares of $b_{j}^{(i)}$. (E.g., $b_{j \mid 1}^{(i)}$ and $b_{j \mid 2}^{(i)}$ are random and $b_{j \mid 3}^{(i)}=b_{j \mid 1}^{(i)} \oplus b_{j \mid 2}^{(i)} \oplus b_{j}^{(i)}$.)

3. Let $(p k, s k) \leftarrow \operatorname{Gen}\left(1^{k}\right)$. For $0 \leq i \leq m$, and $j, j^{\prime} \in\{1,2,3\}$, let $\sigma_{j \mid j^{\prime}}^{(i)}=$ $\operatorname{Sign}_{s k}\left(i\|j\| j^{\prime} \| b_{j \mid j^{\prime}}^{(i)}\right)$.

\section{Output:}

1. Send to each $P_{j}$ the public key $p k$ and the values $\left\{\left(b_{1 \mid j}^{(i)}, \sigma_{1 \mid j}^{(i)}\right),\left(b_{2 \mid j}^{(i)}, \sigma_{2 \mid j}^{(i)}\right),\left(b_{3 \mid j}^{(i)}, \sigma_{3 \mid j}^{(i)}\right)\right\}_{i=0}^{m}$. Additionally, for each $j \in\{1,2,3\}$ parties $P_{j-1}$ and $P_{j+1}$ receive the value $b_{j \mid j}^{(0)}$.

Fig. 1. Functionality ShareGen

If a single party $P_{j}$ aborts in some iteration $i$, then the remaining players $P_{j-1}$ and $P_{j+1}$ jointly reconstruct the value $b_{j}^{(i-1)}$ and output this value. (These two parties jointly have enough information to do this.) If two parties abort in some iteration $i$ (whether at the same time, or one after the other) then the remaining party simply outputs its own input.

We refer to Fig. 1 and Fig. [2 for the formal specification of the protocol. We now prove that this protocol securely computes maj with complete fairness.

Theorem 7. Assume that (Gen, Sign, Vrfy) is a secure signature scheme, that $\pi$ securely computes ShareGen with designated abort, and that $\pi_{\mathrm{OR}}$ securely computes OR with complete fairness 5 Then the protocol in Figure 2 securely computes maj with complete fairness.

Proof. Let $\Pi$ denote the protocol of Figure [2. Observe that $\Pi$ yields the correct output with all but negligible probability when all players are honest. This is because, with all but negligible probability, $i^{*} \leq m$, and then $b_{j}^{(m)}=\operatorname{maj}\left(x_{1}, x_{2}, x_{3}\right)$. We thus focus on security of $\Pi$.

\footnotetext{
${ }^{5}$ It is shown in 2 that such a protocol exists under standard assumptions.
} 


\section{Protocol 1}

Inputs: Party $P_{i}$ has input $x_{i} \in\{0,1\}$. The security parameter is $k$.

\section{The protocol:}

\section{Preliminary phase:}

(a) Parties $P_{1}, P_{2}$ and $P_{3}$ run a protocol $\pi$ for computing ShareGen. Each player uses their respective inputs, $x_{1}, x_{2}$ and $x_{3}$, and security parameter $k$.

(b) If $P_{2}$ and $P_{3}$ receive $\perp$ from this execution, then $P_{2}$ and $P_{3}$ run a twoparty protocol $\pi_{\mathrm{OR}}$ to compute the logical-or of their inputs.

Otherwise, continue to the next stage.

In what follows, parties always verify signatures; invalid signatures are treated as an abort.

2. For $i=1, \ldots, m-1$ do:

\section{Broadcast shares:}

(a) Each $P_{j}$ broadcasts $\left(b_{j \mid j}^{(i)}, \sigma_{j \mid j}^{(i)}\right)$.

(b) If (only) $P_{j}$ aborts:

i. $P_{j-1}$ and $P_{j+1}$ broadcast $\left(b_{j \mid j-1}^{(i-1)}, \sigma_{j \mid j-1}^{(i-1)}\right)$ and $\left(b_{j \mid j+1}^{(i-1)}, \sigma_{j \mid j+1}^{(i-1)}\right)$, respectively.

ii. If one of $P_{j-1}, P_{j+1}$ aborts in the previous step, the remaining player outputs its own input value. Otherwise, $P_{j-1}$ and $P_{j+1}$ both output $b_{j}^{(i-1)}=b_{j \mid 1}^{(i-1)} \oplus b_{j \mid 2}^{(i-1)} \oplus b_{j \mid 3}^{(i-1)}$. (Recall that if $i=1$, parties $P_{j-1}$ and $P_{j+1}$ received $b_{j \mid j}^{(0)}$ as output from $\pi$.)

(c) If two parties abort, the remaining player outputs its own input value.

3. In round $\boldsymbol{i}=\boldsymbol{m}$ do:

(a) Each $P_{j}$ broadcasts $b_{1 \mid j}^{(m)}, \sigma_{1 \mid j}^{(m)}$.

(b) If no one aborts, then all players output $b_{1}^{(m)}=b_{1 \mid 1}^{(m)} \oplus b_{1 \mid 2}^{(m)} \oplus b_{1 \mid 3}^{(m)}$. If (only) $P_{j}$ aborts, then $P_{j-1}$ and $P_{j+1}$ proceed as in step 2b If two players abort, the remaining player outputs its own input as in step [2C

Fig. 2. A protocol for computing majority

When no parties are corrupt, security is straightforward since we assume the existence of a private broadcast channel. We therefore consider separately the cases when a single party is corrupted and when two parties are corrupted. Since the entire protocol is symmetric except for the fact that $P_{1}$ may choose to abort $\pi$, without loss of generality we may analyze the case when the adversary corrupts $P_{1}$ and the case when the adversary corrupts $\left\{P_{1}, P_{2}\right\}$. In each case, we prove security of $\Pi$ in a hybrid world where there is an ideal functionality computing ShareGen (with abort) as well as an ideal functionality computing OR (with complete fairness). Applying the composition theorem of [9] then gives the desired result. A proof for the case where only $P_{1}$ is corrupted turns out to be fairly straightforward, and is given in Appendix A.1.

Claim 8. For every non-uniform, poly-time adversary $\mathcal{A}$ corrupting $P_{1}$ and $P_{2}$ and running $\Pi$ in a hybrid model with access to ideal functionalities 
computing ShareGen (with abort) and OR (with completes fairness), there exists a non-uniform, poly-time adversary $\mathcal{S}$ corrupting $P_{1}$ and $P_{2}$ and running in the ideal world with access to an ideal functionality computing maj (with complete fairness), such that

$$
\begin{aligned}
& \left\{\operatorname{IDEAL}_{\text {maj }, \mathcal{S}}\left(x_{1}, x_{2}, x_{3}, k\right)\right\}_{x_{i} \in\{0,1\}, k \in \mathbb{N}} \\
& \stackrel{\mathrm{s}}{=}\left\{\operatorname{HYBRID}_{\Pi, \mathcal{A}}^{\text {ShareGen, OR }}\left(x_{1}, x_{2}, x_{3}, k\right)\right\}_{x_{i} \in\{0,1\}, k \in \mathbb{N}}
\end{aligned}
$$

Proof. This case is significantly more complex than the case when only a single party is corrupted, since here $\mathcal{A}$ learns $b_{3}^{(i)}$ in each iteration $i$ of the second phase. As in [2, we must deal with the fact that $\mathcal{A}$ might abort exactly in iteration $i^{*}$, after learning the correct output but before $P_{3}$ has enough information to compute the correct output.

We describe a simulator $\mathcal{S}$ who corrupts $P_{1}$ and $P_{2}$ and runs $\mathcal{A}$ as a black-box. For ease of exposition in what follows, we refer to the actions of $P_{1}$ and $P_{2}$ when more formally we mean the action of $\mathcal{A}$ on behalf of those parties.

1. $\mathcal{S}$ invokes $\mathcal{A}$ on the inputs $x_{1}$ and $x_{2}$, the auxiliary input $z$, and the security parameter $k$.

2. $\mathcal{S}$ receives $x_{1}^{\prime}$ and $x_{2}^{\prime}$ from $P_{1}$ and $P_{2}$, respectively, as input to ShareGen. If $x_{1}^{\prime} \notin\{0,1\}$ (resp., $x_{2}^{\prime} \notin\{0,1\}$ ), then $\mathcal{S}$ sets $x_{1}^{\prime}=1$ (resp., $x_{2}^{\prime}=1$ ).

3. $\mathcal{S}$ computes $(s k, p k) \leftarrow \operatorname{Gen}\left(1^{k}\right)$, and then generates shares as follows:

(a) Choose $\left\{b_{1 \mid 1}^{(i)}, b_{2 \mid 1}^{(i)}, b_{3 \mid 1}^{(i)}, b_{1 \mid 2}^{(i)}, b_{2 \mid 2}^{(i)}, b_{3 \mid 2}^{(i)}\right\}_{i=0}^{m}$ uniformly at random.

(b) Choose $\hat{x}_{3} \leftarrow\{0,1\}$ and set $b_{3}^{(0)}=\operatorname{maj}\left(x_{1}^{\prime}, x_{2}^{\prime}, \hat{x}_{3}\right)$. Set $b_{3 \mid 3}^{(0)}=b_{3}^{(0)} \oplus b_{3 \mid 1}^{(0)} \oplus$ $b_{3 \mid 2}^{(0)}$.

$\mathcal{S}$ then hands $\mathcal{A}$ the public key $p k$, the values $\left\{b_{1 \mid 1}^{(i)}, b_{2 \mid 1}^{(i)}, b_{3 \mid 1}^{(i)}, b_{1 \mid 2}^{(i)}, b_{2 \mid 2}^{(i)}, b_{3 \mid 2}^{(i)}\right\}_{i=0}^{m}$ (along with their appropriate signatures), and the value $b_{3 \mid 3}^{(0)}$ as the outputs of $P_{1}$ and $P_{2}$ from ShareGen.

4. If $P_{1}$ aborts execution of ShareGen, then $\mathcal{S}$ extracts $x_{2}^{\prime \prime}$ from $P_{2}$ as its input to OR. It then sends $\left(1, x_{2}^{\prime \prime}\right)$ to the trusted party computing maj, outputs whatever $\mathcal{A}$ outputs, and halts.

5. Otherwise, if $P_{1}$ does not abort, then $\mathcal{S}$ picks a value $i^{*}$ according to a geometric distribution with parameter $\alpha=\frac{1}{5}$.

In what follows, for ease of description, we will use $x_{1}$ and $x_{2}$ in place of $x_{1}^{\prime}$ and $x_{2}^{\prime}$, keeping in mind that that $\mathcal{A}$ could of course have used substituted inputs. We also ignore the presence of signatures from now on, and leave the following implicit in what follows: (1) $\mathcal{S}$ always computes an appropriate signature when sending any value to $\mathcal{A} ;(2) \mathcal{S}$ treats an incorrect signature as an abort; and (3) if $\mathcal{S}$ ever receives a valid signature on a previously unsigned message (i.e., a forgery), then $\mathcal{S}$ outputs fail and halts.

Also, from here on we will say that $\mathcal{S}$ sends $b$ to $\mathcal{A}$ in round $i$ if $\mathcal{S}$ sends a value $b_{3 \mid 3}^{(i)}$ such that $b_{3 \mid 3}^{(i)} \oplus b_{3 \mid 1}^{(i)} \oplus b_{3 \mid 2}^{(i)}=b_{3}^{(i)}=b$. 
6. For round $i=1, \ldots, i^{*}-1$, the simulator $\mathcal{S}$ computes and then sends $b_{3}^{(i)}$ as follows:

(a) Select $\hat{x}_{3} \leftarrow\{0,1\}$ at random.

(b) $b_{3}^{(i)}=\operatorname{maj}\left(x_{1}, x_{2}, \hat{x}_{3}\right)$.

7. If $P_{1}$ aborts in round $i<i^{*}$, then $\mathcal{S}$ sets $\hat{x}_{2}=x_{2}$ and assigns a value to $\hat{x}_{1}$ according to the following rules that depend on the values of $\left(x_{1}, x_{2}\right)$ and on the value of $b_{3}^{(i)}$ :

(a) If $x_{1}=x_{2}$, then $\mathcal{S}$ sets $\hat{x}_{1}=x_{1}$ with probability $\frac{3}{8}$ (and sets $\hat{x}_{1}=\bar{x}_{1}$ otherwise).

(b) If $x_{1} \neq x_{2}$ and $b_{3}^{(i)}=x_{1}$, then $\mathcal{S}$ sets $\hat{x}_{1}=x_{1}$ with probability $\frac{1}{4}$ (and sets $\hat{x}_{1}=\bar{x}_{1}$ otherwise).

(c) If $x_{1} \neq x_{2}$ and $b_{3}^{(i)}=x_{2}$, then $\mathcal{S}$ sets $\hat{x}_{1}=x_{1}$ with probability $\frac{1}{2}$ (and sets $\hat{x}_{1}=\bar{x}_{1}$ otherwise).

$\mathcal{S}$ then finishes the simulation as follows:

(a) If $\hat{x}_{1} \neq \hat{x}_{2}$, then $\mathcal{S}$ submits $\left(\hat{x}_{1}, \hat{x}_{2}\right)$ to the trusted party computing maj. Denote the output it receives from the trusted party by $b_{\text {out }}$. Then $\mathcal{S}$ sets $b_{1}^{(i-1)}=b_{\text {out }}$, computes $b_{1 \mid 3}^{(i-1)}=b_{1}^{(i-1)} \oplus b_{1 \mid 1}^{(i-1)} \oplus b_{1 \mid 2}^{(i-1)}$, sends $b_{1 \mid 3}^{(i-1)}$ to $P_{2}$ (on behalf of $P_{3}$ ), outputs whatever $\mathcal{A}$ outputs, and halts.

(b) If $\hat{x}_{1}=\hat{x}_{2}$, then $\mathcal{S}$ sets $b_{1}^{(i-1)}=\hat{x}_{1}=\hat{x}_{2}$, computes $b_{1 \mid 3}^{(i-1)}=b_{1}^{(i-1)} \oplus$ $b_{1 \mid 1}^{(i-1)} \oplus b_{1 \mid 2}^{(i-1)}$, and sends $b_{1 \mid 3}^{(i-1)}$ to $P_{2}$ (on behalf of $P_{3}$ ). (We stress that this is done before sending anything to the trusted party computing maj.) If $P_{2}$ aborts, then $\mathcal{S}$ sends $(0,1)$ to the trusted party computing maj. Otherwise, it sends $\left(\hat{x}_{1}, \hat{x}_{2}\right)$ to the trusted party computing maj. In both cases it outputs whatever $\mathcal{A}$ outputs, and then halts.

If $P_{2}$ aborts in round $i<i^{*}$, then $\mathcal{S}$ acts analogously but swapping the roles of $P_{1}$ and $P_{2}$ as well as $x_{1}$ and $x_{2}$.

If both parties abort in round $i<i^{*}$ (at the same time), then $\mathcal{S}$ sends $(0,1)$ to the trusted party computing maj, outputs whatever $\mathcal{A}$ outputs, and halts.

8. In round $i^{*}$ :

(a) If $x_{1} \neq x_{2}$, then $\mathcal{S}$ submits $\left(x_{1}, x_{2}\right)$ to the trusted party. Let $b_{\text {out }}=$ $\operatorname{maj}\left(x_{1}, x_{2}, x_{3}\right)$ denote the output.

(b) If $x_{1}=x_{2}$, then $\mathcal{S}$ simply sets $b_{\text {out }}=x_{1}=x_{2}$ without querying the trusted party and continues. (Note that in this case, $b_{\text {out }}=\operatorname{maj}\left(x_{1}, x_{2}, x_{3}\right)$ even though $\mathcal{S}$ did not query the trusted party.)

9. In rounds $i^{*}, \ldots, m-1$, the simulator $\mathcal{S}$ sends $b_{\text {out }}$ to $\mathcal{A}$.

If $\mathcal{A}$ aborts $P_{1}$ and $P_{2}$ simultaneously, then $\mathcal{S}$ submits $(1,0)$ to the trusted party (if he hasn't already done so in step $8 \mathrm{a}$ ), outputs whatever $\mathcal{A}$ outputs, and halts.

If $\mathcal{A}$ aborts $P_{1}$ (only), then $\mathcal{S}$ sets $b_{1}^{(i-1)}=b_{\text {out }}$, computes $b_{1 \mid 3}^{(i-1)}=$ $b_{1}^{(i-1)} \oplus b_{1 \mid 1}^{(i-1)} \oplus b_{1 \mid 2}^{(i-1)}$, and sends $b_{1 \mid 3}^{(i-1)}$ to $P_{2}$ (on behalf of $P_{3}$ ). Then:

Case 1: $\boldsymbol{x}_{\mathbf{1}} \neq \boldsymbol{x}_{\mathbf{2}}$. Here $\mathcal{S}$ has already sent $\left(x_{1}, x_{2}\right)$ to the trusted party. So $\mathcal{S}$ simply outputs whatever $\mathcal{A}$ outputs and ends the simulation.

Case 2: $\boldsymbol{x}_{\mathbf{1}}=\boldsymbol{x}_{\mathbf{2}}$. If $P_{2}$ does not abort, then $\mathcal{S}$ sends $\left(x_{1}, x_{2}\right)$ to the trusted party. If $P_{2}$ aborts, then $\mathcal{S}$ sends $(0,1)$ to the trusted party. In both cases $\mathcal{S}$ then outputs whatever $\mathcal{A}$ outputs and halts. 
If $\mathcal{A}$ aborts $P_{2}$ (only), then $\mathcal{S}$ acts as above but swapping the roles of $P_{1}, P_{2}$ and $x_{1}, x_{2}$. If $\mathcal{A}$ does not abort anyone through round $m$, then $\mathcal{S}$ sends $\left(x_{1}, x_{2}\right)$ to the trusted party (if he hasn't already done so), outputs what $\mathcal{A}$ outputs, and halts.

The above constitutes the full description of $\mathcal{S}$. Due to space limitations, the analysis of $\mathcal{S}$ is given in the full version of this paper [].

\section{Completely-Fair Computation of Boolean OR}

The protocol in the previous section enables completely-fair computation of 3party majority; unfortunately, we were not able to extend the approach to the case of $n>3$ parties. In this section, we demonstrate feasibility of completelyfair computation of a non-trivial function for an arbitrary number of parties $n$, any $t<n$ of whom are corrupted. Specifically, we show how to compute boolean OR with complete fairness.

The basic idea behind our protocol is to have the parties repeatedly try to compute OR on committed inputs using a protocol that is secure-withdesignated-abort where only the lowest-indexed party can force an abort. (See the full version [7 for further discussion.) The key observation is that in case of an abort, the dishonest players only "learn something" about the inputs of the honest players in case all the malicious parties use input 0 . (If any of the malicious players holds input 1 , then the output is always 1 regardless of the inputs of the honest parties.) So, if the lowest-indexed party is corrupt and aborts the computation of the committed OR, then the remaining parties simply recompute the committed OR using ' 0 ' as the effective input for any parties who have already been eliminated. The parties repeatedly proceed in this fashion, eliminating dishonest parties at each iteration. Eventually, when the lowest-indexed player is honest, the process terminates and all honest players receive (correct) output.

The actual protocol follows the above intuition, but is a bit more involved. A formal description of the protocol is given in Fig. 3. and the "committed OR" functionality is defined in Fig. 4.

Theorem 9. Assume Com is a computationally-hiding, statistically-binding commitment scheme, and that $\pi_{\mathcal{P}}$ securely computes CommittedOR $\mathrm{R}_{\mathcal{P}}$ (with abort). Then the protocol of Fig. 3 computes OR with complete fairness.

Proof. Let $\Pi$ denote the protocol of Fig. 3. For simplicity we assume Com is perfectly binding, though statistical binding suffices. For any non-uniform, polynomial time adversary $\mathcal{A}$ in the hybrid world, we demonstrate a non-uniform polynomial-time adversary $\mathcal{S}$ corrupting the same parties as $\mathcal{A}$ and running in the ideal world with access to an ideal functionality computing OR (with complete fairness), such that

$$
\begin{aligned}
& \left\{\operatorname{IDEALOR}, \mathcal{S}\left(x_{1}, \ldots, x_{n}, k\right)\right\}_{x_{i} \in\{0,1\}, k \in \mathbb{N}} \\
& \stackrel{\mathrm{c}}{\equiv}\left\{\operatorname{HYBRID}_{\Pi, \mathcal{A}}^{\text {CommittedOR }}\left(x_{1}, \ldots, x_{n}, k\right)\right\}_{x_{i} \in\{0,1\}, k \in \mathbb{N}} .
\end{aligned}
$$




\section{Protocol 2}

Inputs: Each party $P_{i}$ holds input $x_{i} \in\{0,1\}$, and the security parameter is $k$.

\section{Computation:}

1. Let $\mathcal{P}=\left\{P_{1}, \ldots, P_{n}\right\}$ be the set of all players.

2. Each player $P_{i}$ chooses random coins $r_{i}$ and broadcasts $c_{i}=\operatorname{Com}\left(1^{k}, x_{i}, r_{i}\right)$, where Com denotes a computationally-hiding, statistically-binding commitment scheme. If any party $P_{i}$ does not broadcast anything (or otherwise broadcasts an invalid value), then all honest players output 1 . Otherwise, let $\boldsymbol{c}=\left(c_{1}, \ldots, c_{n}\right)$.

3. All players $P_{i} \in \mathcal{P}$ run a protocol $\pi_{\mathcal{P}}$ for computing CommittedOR Co $_{\mathcal{P}}$, with party $P_{i}$ using $\left(x_{i}, r_{i}, \boldsymbol{c}_{\mathcal{P}}\right)$ as its input where $\boldsymbol{c}_{\mathcal{P}} \stackrel{\text { def }}{=}\left(c_{i}\right)_{i: P_{i} \in \mathcal{P}}$.

4. If players receive $\perp$ from the execution of CommittedOR $\operatorname{P}_{\mathcal{P}}$, they set $\mathcal{P}=$ $\mathcal{P} \backslash\left\{P^{*}\right\}$, where $P^{*} \in \mathcal{P}$ is the lowest-indexed player in $\mathcal{P}$, and return to Step $\mathbf{B}$

5. If players receive a set $\mathcal{D} \subset \mathcal{P}$ from the execution of CommittedOR $R_{\mathcal{P}}$, they set $\mathcal{P}=\mathcal{P} \backslash \mathcal{D}$ and return to Step B]

6. If players receive a binary output from the execution of CommittedOR $R_{\mathcal{P}}$, they output this value and end the protocol.

Fig. 3. A protocol computing OR for $n$ players

\section{CommittedOR $_{\mathcal{P}}$}

Inputs: The functionality is run by parties in $\mathcal{P}$. Let the input of player $P_{i} \in \mathcal{P}$ be $\left(x_{i}, r_{i}, \boldsymbol{c}^{i}\right)$ where $\boldsymbol{c}^{i}=\left(c_{j}^{i}\right)_{j: P_{j} \in \mathcal{P}}$. The security parameter is $k$.

For each party $P_{i} \in \mathcal{P}$, determine its output as follows:

1. Say $P_{j}$ disagrees with $P_{i}$ if either (1) $\boldsymbol{c}^{j} \neq \boldsymbol{c}^{i}$ or (2) $\operatorname{Com}\left(1^{k}, x_{j}, r_{j}\right) \neq c_{j}^{i}$. (Note that disagreement is not a symmetric relation.)

2. Let $\mathcal{D}_{i}$ be the set of parties who disagree with $P_{i}$.

3. If there exist any parties that disagree with each other, return $\mathcal{D}_{i}$ as output to $P_{i}$. Otherwise, return $\bigvee_{j: P_{j} \in \mathcal{P}} x_{j}$ to all parties.

Fig. 4. Functionality Committed $\mathrm{OR}_{\mathcal{P}}$, parameterized by a set $\mathcal{P}$

Applying the composition theorem of [9] then proves the theorem.

When no players are corrupt, security is trivial due to the assumed existence of a private broadcast channel. We now describe the execution of $\mathcal{S}$ assuming a set $\mathcal{C} \neq \emptyset$ of corrupted parties:

1. Let $\mathcal{H}=\left\{P_{1}, \ldots P_{n}\right\} \backslash \mathcal{C}$ denote the honest players. Initialize $\mathcal{I}=\mathcal{C}$. Looking ahead, $\mathcal{I}$ denotes the set of corrupted parties who have not yet been eliminated from the protocol.

2. $\mathcal{S}$ invokes $\mathcal{A}$ on the inputs $\left\{x_{i}\right\}_{i: P_{i} \in \mathcal{C}}$, the auxiliary input $z$, and the security parameter $k$. 
3. For $P_{i} \in \mathcal{H}$, the simulator $\mathcal{S}$ gives to $\mathcal{A}$ a commitment $c_{i}=\operatorname{Com}\left(1^{k}, x_{i}, r_{i}\right)$ to $x_{i}=0$ using randomness $r_{i}$. $\mathcal{S}$ then records the commitment $c_{i}$ that is broadcast by $\mathcal{A}$ on behalf of each party $P_{i} \in \mathcal{C}$. If any corrupted player fails to broadcast a value $c_{i}$, then $\mathcal{S}$ submits 1's to the trusted party on behalf of all corrupted parties, outputs whatever $\mathcal{A}$ outputs, and halts.

4. If $\mathcal{I}=\emptyset, \mathcal{S}$ submits (on behalf of all the corrupted parties) 0's to the trusted party computing OR (unless it has already done so). It then outputs whatever $\mathcal{A}$ outputs, and halts. If $\mathcal{I} \neq \emptyset$, continue to the next step.

5. $\mathcal{S}$ sets $\mathcal{P}=\mathcal{H} \cup \mathcal{I}$ and obtains inputs $\left\{\left(r_{i}, x_{i}, c^{i}\right)\right\}_{i: P_{i} \in \mathcal{I}}$ for the computation of CommittedOR $\mathrm{R}_{\mathcal{P}}$. For each $P_{i} \in \mathcal{P}$, the simulator $\mathcal{S}$ computes the list of players $\mathcal{D}_{i}$ that disagree with $P_{i}$ (as in Fig. 目), using as the inputs of the honest parties the commitments defined in Step [3 and assuming that honest parties provide correct decommitments. Observe that if $P_{i}, P_{j} \in \mathcal{H}$ then $\mathcal{D}_{i}=\mathcal{D}_{j} \subseteq \mathcal{I}$. Let $\mathcal{D}_{\mathcal{H}} \subseteq \mathcal{I}$ be the set of parties that disagree with the honest parties.

Let $P^{*}$ be the lowest-indexed player in $\mathcal{P}$. If no parties disagree with each other, go to Step [6] Otherwise:

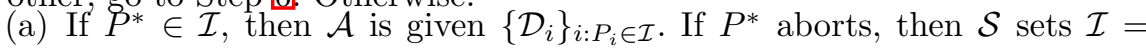
$\mathcal{I} \backslash\left\{P^{*}\right\}$ and goes to Step 4 If $P^{*}$ does not abort, then $\mathcal{S}$ sets $\mathcal{I}=\mathcal{I} \backslash \mathcal{D}_{\mathcal{H}}$ and goes to Step 四

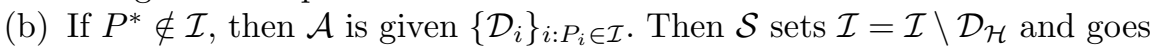
to Step 目

6. $\mathcal{S}$ computes the value $b=\bigvee_{P_{i} \in \mathcal{I}} x_{i}$.

(a) If $b=0$, and $\mathcal{S}$ has not yet queried the trusted party computing OR, then $\mathcal{S}$ submits 0's (on behalf of all the corrupted parties) to the trusted party and stores the output of the trusted party as $b_{\text {out }}$. $\mathcal{S}$ gives $b_{\text {out }}$ to $\mathcal{A}$ (either as just received from the trusted party, or as stored in a previous execution of this step).

(b) If $b=1$, then $\mathcal{S}$ gives the value 1 to $\mathcal{A}$ without querying the trusted party.

$\mathcal{S}$ now continues as follows:

(a) If $P^{*} \in \mathcal{I}$ and $P^{*}$ aborts, then $\mathcal{S}$ sets $\mathcal{I}=\mathcal{I} \backslash\left\{P^{*}\right\}$ and goes to Step 4 .

(b) If $P^{*} \notin \mathcal{I}$, or if $P^{*}$ does not abort, then $\mathcal{S}$ submits 1 's to the trusted party if it has not yet submitted 0's. It outputs whatever $\mathcal{A}$ outputs, and halts.

We refer the reader to the full version of this paper [7] for an analysis of the above simulation.

\section{References}

1. Cleve, R.: Limits on the security of coin flips when half the processors are faulty. In: Proc. 18th Annual ACM Symposium on Theory of Computing (STOC), pp. 364-369 (1986)

2. Gordon, D., Hazay, C., Katz, J., Lindell, Y.: Complete fairness in secure two-party computation. In: Proc. 40th Annual ACM Symposium on Theory of Computing (STOC) (2008) 
3. Chor, B., Ishai, Y.: On privacy and partition arguments. Inf. Comput. 167(1), 2-9 (2001)

4. Kilian, J., Kushilevitz, E., Micali, S., Ostrovsky, R.: Reducibility and completeness in private computations. SIAM J. Computing 29(4), 1189-1208 (2000)

5. Chor, B., Kushilevitz, E.: A zero-one law for boolean privacy. SIAM Journal of Discrete Math 4(1), 36-47 (1991)

6. Ishai, Y., Kushilevitz, E., Lindell, Y., Petrank, E.: On combining privacy with guaranteed output delivery in secure multiparty computation. In: Dwork, C. (ed.) CRYPTO 2006. LNCS, vol. 4117, pp. 483-500. Springer, Heidelberg (2006)

7. Gordon, S.D., Katz, J.: Complete fairness in multi-party computation without an honest majority. In: 6th Theory of Cryptography Conference, TCC (2009), http://eprint.iacr.org/2008/458

8. Goldreich, O.: Foundations of Cryptography, Basic Applications, vol. 2. Cambridge University Press, Cambridge (2004)

9. Canetti, R.: Security and composition of multiparty cryptographic protocols. Journal of Cryptology 13(1), 143-202 (2000)

\section{A Proofs}

\section{A.1 Proof of Security for Majority with a Single Corrupted Party}

Claim 10. For every non-uniform, polynomial-time adversary $\mathcal{A}$ corrupting $P_{1}$ and running $\Pi$ in a hybrid model with access to ideal functionalities computing ShareGen (with abort) and OR (with complete fairness), there exists a nonuniform, poly-time adversary $\mathcal{S}$ corrupting $P_{1}$ and running in the ideal world with access to an ideal functionality computing maj (with complete fairness), such that

$$
\begin{aligned}
& \left\{\operatorname{IDEAL}_{\text {maj }, \mathcal{S}}\left(x_{1}, x_{2}, x_{3}, k\right)\right\}_{x_{i} \in\{0,1\}, k \in \mathbb{N}} \\
& \stackrel{\mathrm{s}}{=}\left\{\operatorname{HYBRID}_{\Pi, \mathcal{A}}^{\text {ShareGen, OR }}\left(x_{1}, x_{2}, x_{3}, k\right)\right\}_{x_{i} \in\{0,1\}, k \in \mathbb{N}} .
\end{aligned}
$$

Proof. Fix some polynomial-time adversary $\mathcal{A}$ corrupting $P_{1}$. We now describe a simulator $\mathcal{S}$ that also corrupts $P_{1}$ and runs $\mathcal{A}$ as a black box.

1. $\mathcal{S}$ invokes $\mathcal{A}$ on the input $x_{1}$, the auxiliary input $z$, and the security parameter $k$.

2. $\mathcal{S}$ receives input $x_{1}^{\prime} \in\{0,1\}$ on behalf of $P_{1}$ as input to ShareGen.

3. $\mathcal{S}$ computes $(s k, p k) \leftarrow \operatorname{Gen}\left(1^{k}\right)$, and gives to $\mathcal{A}$ the public key $p k$ and values $b_{2 \mid 2}^{(0)}, b_{3 \mid 3}^{(0)}$, and $\left\{b_{1 \mid 1}^{(i)}, b_{2 \mid 1}^{(i)}, b_{3 \mid 1}^{(i)}\right\}_{i=0}^{m}$ (along with their appropriate signatures) chosen uniformly at random.

4. If $\mathcal{A}$ aborts execution of ShareGen, then $\mathcal{S}$ sends 1 to the trusted party computing maj, outputs whatever $\mathcal{A}$ outputs, and halts. Otherwise, $\mathcal{S}$ picks a value $i^{*}$ according to a geometric distribution with parameter $\alpha=\frac{1}{5}$.

For simplicity in what follows, we ignore the presence of signatures and leave the following implicit from now on: (1) $\mathcal{S}$ always computes an appropriate signature when sending any value to $\mathcal{A} ;(2) \mathcal{S}$ treats an incorrect signature as an abort; and (3) if $\mathcal{S}$ ever receives a valid signature on a previously unsigned message, then $\mathcal{S}$ outputs fail and halts. 
5. $\mathcal{S}$ now simulates the rounds of the protocol one-by-one: for $i=1$ to $m-1$, the simulator chooses random $b_{2 \mid 2}^{(i)}$ and $b_{3 \mid 3}^{(i)}$ and sends these to $\mathcal{A}$. During this step, an abort by $\mathcal{A}$ (on behalf of $P_{1}$ ) is treated as follows:

(a) If $P_{1}$ aborts in round $i \leq i^{*}$, then $\mathcal{S}$ chooses a random value $\hat{x}_{1}$ and sends it to the trusted party computing maj.

(b) If $P_{1}$ aborts in round $i>i^{*}$, then $\mathcal{S}$ submits $x_{1}^{\prime}$ to the trusted party computing maj.

In either case, $\mathcal{S}$ then outputs whatever $\mathcal{A}$ outputs and halts.

6. If $P_{1}$ has not yet aborted, $\mathcal{S}$ then simulates the final round of the protocol. $\mathcal{S}$ sends $x_{1}^{\prime}$ to the trusted party, receives $b_{\text {out }}=\operatorname{maj}\left(x_{1}^{\prime}, x_{2}, x_{3}\right)$, and chooses $b_{1 \mid 2}^{(m)}$ and $b_{1 \mid 3}^{(m)}$ at random subject to $b_{1 \mid 2}^{(m)} \oplus b_{1 \mid 3}^{(m)} \oplus b_{1 \mid 1}^{(m)}=b_{\text {out }} \mathcal{S}$ then gives these values to $\mathcal{A}$, outputs whatever $\mathcal{A}$ outputs, and halts.

Due to the security of the underlying signature scheme, the probability that $\mathcal{S}$ outputs fail is negligible in $k$. Note that the view of $P_{1}$ is otherwise statistically close in both worlds. Indeed, until round $m$ the view of $P_{1}$ is independent of the inputs of the other parties in both the real and ideal worlds. In round $m$ itself, $P_{1}$ learns the (correct) output $b_{\text {out }}$ in the ideal world and learns this value with all but negligible probability in the real world.

We therefore only have to argue that outputs of the two honest parties in the real and ideal worlds are statistically close. Clearly this is true if $P_{1}$ never aborts. As for the case when $P_{1}$ aborts at some point during the protocol, we divide our analysis into the following cases:

- If $P_{1}$ aborts the execution of ShareGen in step 4 then $\mathcal{S}$ submits ' 1 ' on behalf of $P_{1}$ to the trusted party computing maj. Thus, in the ideal world, the outputs of $P_{2}$ and $P_{3}$ will be $\operatorname{maj}\left(1, x_{2}, x_{3}\right)$. In the real world, if $P_{1}$ aborts computation of ShareGen, the honest parties output $\mathrm{OR}\left(x_{2}, x_{3}\right)$. Since $\operatorname{maj}\left(1, x_{2}, x_{3}\right)=\mathrm{OR}\left(x_{2}, x_{3}\right)$, their outputs are the same.

- If $P_{1}$ aborts in round $i$ of the protocol (cf. step [5), then in both the real and ideal worlds the following holds:

- If $i \leq i^{*}$, then $P_{2}$ and $P_{3}$ output $\operatorname{maj}\left(\hat{x}_{1}, x_{2}, x_{3}\right)$ where $\hat{x}_{1}$ is chosen uniformly at random.

- If $i>i^{*}$, then $P_{2}$ and $P_{3}$ output $\operatorname{maj}\left(x_{1}^{\prime}, x_{2}, x_{3}\right)$

Since $i^{*}$ is identically distributed in both worlds, the outputs of $P_{2}$ and $P_{3}$ in this case are identically distributed as well.

- If $P_{1}$ aborts in round $m$ (cf. step [6), then in the ideal world the honest parties will output maj $\left(x_{1}^{\prime}, x_{2}, x_{3}\right)$. In the real world the honest parties output $\operatorname{maj}\left(x_{1}^{\prime}, x_{2}, x_{3}\right)$ as long as $i^{*} \leq m-1$, which occurs with all but negligible probability.

This completes the proof. 